\title{
Radiologic Clinics of North America: Imaging of Lymphoma
}

J. Rademaker, ed.

Philadelphia, PA: Saunders, 2008, 436 pages, $\$ 89$

Lymphomas are the most common hematologic malignancies in the United States, accounting for up to 5\% of all new cancer cases. As a group, they are the fifth leading cause of cancer deaths. Since the landmark report released in 1832 by Thomas Hodgkin, the classification of lymphomas has undergone repetitive adjustment, primarily a reflection of novel clinical and basic science discoveries. The more recent classification systems include the International Working Formula developed in 1982, the Revised European-American Lymphoma (REAL) system, and the current World Health Organization system, which builds on the REAL system by incorporating genetic and immunophenotypic information. Paralleling the developments in the classification system, the diagnosis, treatment, and follow-up of lymphoma continues to adapt and evolve. In light of the accelerated pace of change, this book serves as a comprehensive overview of the most current radiologic and clinical aspects of this diverse disease.

Released by the Radiologic Clinics of North America, this book is a single issue of a radiologic volume covering a spectrum of diseases, systems, and modalities. It is an exhaustive collection of 14 review articles covering a range of lymphoma topics, with a primary focus on imaging techniques and standards. The book serves as a source of the most current guidelines for the diagnosis, treatment, and radiologic follow-up of lymphomas. Although it is primarily directed toward radiologists, as the editor prefaces, this book is a compelling read for all clinicians who encounter patients affected by lymphoma.

The first article appropriately is an overview of the diagnosis and management of lymphoma. The historic development of the modern classification is described. The 2 major types of lymphoma, Hodgkin disease and non-Hodgkin lymphoma, are introduced, and the various subtypes are presented in table format. Like the subsequent articles, emphasis is placed on the most common Hodgkin disease and non-Hodgkin lymphoma subtypes. This article also provides a detailed description of the proper staging of lymphoma. The article effectively breaks down each major subtype of both Hodgkin disease and non-Hodgkin lymphoma, describing the diagnosis, clinical presentation, staging, and management and serving as a foundation for the subsequent articles.

The next group of articles introduces and expounds a major theme of the collective: the central role of ${ }^{18}$ F-FDG PET and
PET/CT in the primary staging, response assessment, and treatment monitoring of lymphomas. The discussion begins with a thorough explanation of PET physics and the fundamental principles of the modality. In the same article, other important discussion points are developed such as the appropriateness of oral and intravenous contrast material during PET/CT and the uptake patterns of common lymphoma subtypes. In the subsequent article, the development of lymphoma staging criteria and their adaptation within the central application of PET and PET/CT is described. Further, the article includes sensitivities and specificities of the various imaging modalities in the primary detection of lymphoma. Lastly, the technical response criteria for lymphoma are described, including the radiologic criteria for complete remission, partial remission, stable disease, and relapsed disease.

The subsequent article describes the potential pitfalls and limitations of PET/CT in the assessment and management of lymphoma. Despite the overwhelming positives of this modality, this article delineates important theoretic and practical considerations. One of the more common problems includes distinguishing residual disease or viable tumor from physiologic variability (e.g., inflammation and infection). Other interesting topics include the necessity of consolidative radiotherapy for certain lymphoma subtypes, the rationale for interim PET scans, and response-adapted therapy modification based on PET.

The following group of articles, which will be of much interest for both the nuclear medicine physician and the radiologist, is a comprehensive imaging guideline of lymphomas in each major anatomic system using the principal imaging modalities, including CT, ultrasound, MRI, fluoroscopic studies, and PET/CT, among others. Most images in this section are cross-sectional CT scans with detailed descriptions that are of particular importance for the nonradiologist learning CT. In addition, this set of articles includes helpful tables for image interpretation, such as normal measurements of the thymus gland on CT based on age. The greatest feature of these articles is the high quality of image detail and wealth of color images covering almost every major subtype of lymphoma in each major system.

The final 2 articles of this book describe the imaging characteristics of complications associated with receiving hematopoietic stem cell transplants and mantle field radiotherapy. Although management of patients who have 
received these therapies has dramatically improved, after successful treatment many of these patients still have increased morbidity and mortality due to the higher risk of infection, chronic health conditions, and secondary cancers. Early detection of these conditions by imaging can lead to an improved long-term outcome. These articles complete the review of all the major radiologic aspects of this broad and diverse disease.

As a collection of review articles, this book is not sequentially structured like conventional textbooks. Redundancy of information exists between articles, particularly epidemiologic and background information. Despite this limitation, each article appropriately builds on the former, serving to emphasize important background information, such as the epidemiologic proportions of lymphomas and their subtypes, the aggressive subtypes versus the indolent, and important prognostic facts. As a particular strength of this book, the images included are well chosen and are of such high quality that they can be used without the text as an imaging atlas for reference. Ultimately, the Radiologic Clinics of North America and the editor successfully produced a comprehensive source to serve nuclear medicine physicians, radiologists, and clinicians with the most contemporary clinical and radiologic guidelines on lymphoma.

\section{Prashant Shrestha}

University of Minnesota

Minneapolis, Minnesota

\section{Robert Matthews}

Stony Brook University Hospital

Stony Brook, New York

DOI: 10.2967/jnumed.109.067561 